\title{
Ship Motion Measurement Using an Inertial Measurement Unit
}

\author{
Nana O. Abankwa, Steven J. Johnston, Mark Scott \& Simon J. Cox \\ Faculty of Engineering and the Environment \\ University of Southampton \\ Southampton, United Kingdom \\ n.abankwa@ soton.ac.uk, sjj698@zepler.org, mark.scott@soton.ac.uk, s.j.cox@soton.ac.uk
}

\begin{abstract}
Traditional methods of measuring a vessel's motion involve the use of bulky sensor units which usually require cables. These devices are not only expensive, but are difficult and time-consuming to install. They are simply not affordable and do not provide owners of vessels with a cost effective way of measuring and monitoring their vessels' motion during operation. Measured vessel motion could be used to deduce the sea state using the wave buoy analogy. This additional information could be crucial in improving operational safety for vessels. If motion monitoring is to be used to help improve a vessel's operational stability and provide operational critical information, cheaper and simpler alternatives are needed. In this paper, we present a novel motion measurement system which uses a Raspberry $P i$ with a wireless module and an inertial measurement unit (IMU) sensor to measure a vessel's motions. The data collected can either be stored locally on the Raspberry Pi or be sent wirelessly to the user. Compared to traditional devices, the Raspberry Pi is cheaper and easier to install. In addition, due to its computing power, some of the post-processing of data, which is traditionally done on a computer separate from the measuring unit, can be done on the Raspberry Pi. For a practical evaluation of using the Raspberry Pi to measure ship motion, it was placed on a fishing vessel for two hours. The IMU used measured nine degrees of freedom, all of which were recorded at $50 \mathrm{~Hz}$ on the Raspberry Pi. Afterwards, the data was taken from the Raspberry Pi wirelessly and analysed using Matlab, firstly to ensure that the right data was collected at the right frequency, and secondly to visualise the data. The evaluation shows that using the Raspberry $\mathbf{P i}$, as an Internet of Things (IoT) device, is a light-weight, cost-effective, and easy-to-install alternative to traditional devices for measuring vessel motion.
\end{abstract}

Keywords-ship motion; waves; raspberry pi; sensor, IoT;

\section{INTRODUCTION}

The flow of wind over the surface of the sea is one of the most important phenomena in the marine environment. This flow results in the formation of wind-generated waves that can travel across large distances. Interactions between individual waves result in the formation of the complex irregular sea. The sea hosts a wide variety of vessels in an equally varying number of industries. Examples of such vessels include small to medium-sized boats for fishing, roll-on/roll-off (RoRo) ships and container ships for passenger and cargo transportation, and yachts and catamarans for recreational/competitive sailing. The operational safety of these vessels can be increased through good knowledge and understanding of the sea state. Insights into the sea state from measured vessel motion can be gained through the wave buoy analogy [1]. The wave buoy analogy considers vessels as wave buoys so that their motions in response to the sea state can be used to estimate sea state parameters and wave spectra [2].

Operational safety is particularly important in the marine environment since the sea hosts the most dangerous job in the United Kingdom (UK): fishing. According to the British Broadcasting Corporation (BBC), "Fishermen in Britain have a one in twenty chance of being killed on the job during the course of their working lives. In 2009, 13 fishermen were lost at sea, up from just 7 the year before" [3] and in 2010, there were 7 serious incidents involving UK fishing vessels as a result of capsizing [4]. In America, fishing is still the most dangerous job even after attempts by the government to improve safety through regulation [5]. All sea-going vessels face safety risks similar to those faced by fishing vessels. With sea conditions constantly changing, it is difficult for personnel on-board to accurately assess the vessel's stability while it is in operation. This is particularly important because there is no inherent method of determining vessel stability during operation. Some available methods of approximating a vessel's stability use a variety of ship dimensions and variables such as the vessel's lightship weight, centres of gravity, loading conditions, and roll period [6]. However, these methods mentioned are neither used in real-time during the vessel's operation nor require the continuous recording of data during the vessel's normal operation. Therefore, deteriorations in vessel stability due to changing weather conditions cannot be determined while the vessel is in use. The safety risks can be reduced through the use of vessel motion monitoring systems [7].

This paper provides a proof-of-concept by discussing an end-to-end deployment of the Raspberry $\mathrm{Pi}$ as a tool for measuring a fishing vessel's motions. The remainder of this paper is organised as follows: Section II is an introduction to the wave spectrum and Section III introduces the concept of IoT as a possible way of improving operation safety at sea. Section IV describes related work, Section V presents the method, Section VI presents the results and evaluates the method used in this study, and Section VII discusses future work. Finally in Section VIII the paper is concluded. 


\section{THE WAVE SPECTRUM}

A lot of valuable information about the sea state can be gained by analysing the wave spectrum. Methods for obtaining the data from which the wave spectrum is deduced include wave rider buoys, satellite measurements, and wave radar systems. Each of these methods have advantages and disadvantages. The advantage of wave rider buoys is that data obtained from them is freely available on the Internet through sources such as the National Oceanic and Atmospheric Administrations (NOAA) National Data Buoy Center (NDBC), the Met Offices Marine Automatic Weather Stations (MAWS) network, the British Oceanographic Data Centre (BODC), and the Centre for Environment, Fisheries and Aquaculture Science (CEFAS). These buoys provide information such as wind speed, wind direction, significant wave height, and dominant wave period. This information is updated at different frequencies depending on the source. For instance the Met Office updates its data hourly. The disadvantage of using wave rider buoys is that they are in a fixed position and therefore provide information on a limited geographical region. As compared to wave rider buoys, satellite measurements and wave radar systems have the advantage of being able to cover a larger region of interest. In addition, they are not fixed in position. However their disadvantages include their high initial costs and more complex computational hardware. Even though wave radar systems provide more information than several wave buoys, they can cost up to four times the price of wave buoys [8].

To obtain the wave spectrum, sometimes known as the energy spectrum, from collected data, one defines the total energy of the sea to be made up of the energies of all the smaller waves that interact to make up the complex irregular sea. This total energy is representative of the intensity of the sea. "Spectral analysis assumes that the sea state can be considered as a combination or superposition of a large number of regular sinusoidal wave components with different frequencies, heights, and directions," [9] as given by equation 1.

$$
\eta(t)=\sum_{n=1}^{\infty} a_{n} \sin \left(2 \pi f_{n} t+\phi_{n}\right)
$$

where; $\eta(t)$ is the surface elevation at time, $t$ at a fixed point, $a_{n}$ is the amplitude of the nth sinusoidal component, $f_{n}$ is the frequency of the nth sinusoidal component, and $\phi_{n}$ is the phase angle of the nth sinusoidal component.

The wave spectrum shows the distribution of the total energy according to the frequencies of the smaller interacting wave components [10]. Wave spectra are obtained by using Fast Fourier Transforms (FFT) to convert observations of wave height from the time-domain to the frequency domain. Analysis of data recorded in the North Atlantic Ocean have resulted in a number of spectra, each with its own equation to predict the spectral density values using variables such as the significant wave height, significant wave period, and wind speed. Examples of these include the Pierson-Moskowitz spectrum, the Joint North Sea Wave Observation Project (JONSWAP) spectrum, the Bretschneider spectrum, and the International Ship Structures Congress (ISSC) spectrum. Figure 1 [11] shows examples of the JONSWAP and Pierson-Moskowitz spectra.

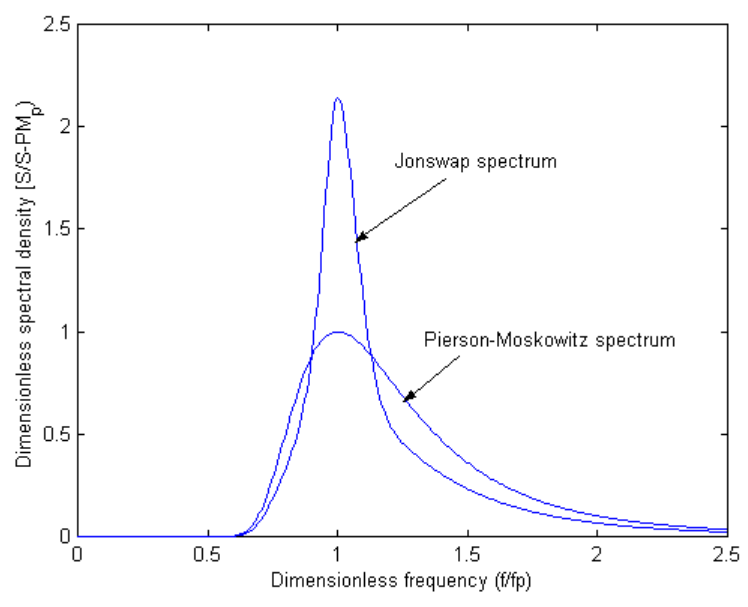

Fig. 1. The Pierson-Moskowitz and JONSWAP spectra

Even though there are variations in the shapes of wave spectra due to different sea states and the type of spectra, for instance whether JONSWAP or ISSC, measurements of wave height follow a Rayleigh distribution as seen in figures 1 and 2. "A Rayleigh distribution is often observed when the overall magnitude of a vector is related to its directional components" [12]. Therefore even though both wind speed and wind direction may be Gaussian variables, their combined effect on the sea surface to generate wind waves follows a Rayleigh distribution. From this distribution of wave heights, even thought the actual sea surface waves cannot be exactly predicted, one can deduce, through statistical methods, important sea state parameters such as the significant wave height $\left(H_{s}\right)$ as seen in figure 2 [13].

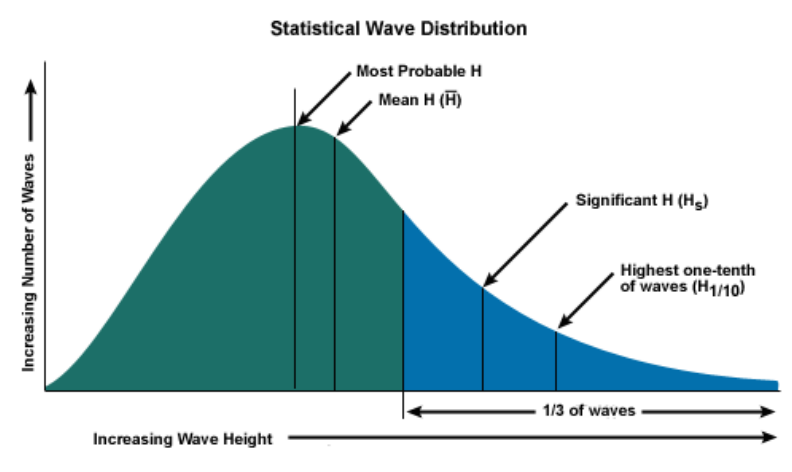

Fig. 2. Statistical wave distribution

\section{INTERNET OF THINGS (IOT)}

IoT presents an alternative open source and low-cost solution to the problem of obtaining measurements about the 
TABLE I

COMPARISON OF PROTOTYPING PLATFORMS

\begin{tabular}{|l|l|l|l|}
\hline & Arduino & Gadgeteer & Raspberry Pi \\
\hline Model & R3 & FEZ Spider & Model B \\
\hline Price & $\$ 30$ & $\$ 120$ & $\$ 35$ \\
\hline Processor & ATMega 328 & ARM7 & ARM11 \\
\hline Clock Speed & $16 \mathrm{MHz}$ & $72 \mathrm{MHz}$ & $700 \mathrm{MHz}$ \\
\hline RAM & $2 \mathrm{MB}$ & $16 \mathrm{MB}$ & $256 \mathrm{MB}$ \\
\hline Flash & $32 \mathrm{~KB}$ & $4.5 \mathrm{MB}$ & (SD CARD) \\
\hline Min Power & $42 \mathrm{~mA}$ & $160 \mathrm{~mA}$ & $700 \mathrm{~mA}$ \\
\hline Dev IDE & Arduino Tool & Visual Studio & IDLE, Linux etc \\
\hline
\end{tabular}

sea state. This can be achieved by using low-cost sensors to measure vessel motion, from which the sea state is deduced, satisfying the definition of IoT [14]. Also, previous work by Giacobbe has shown that a network of sensors in the marine environment being managed with an appropriate software platform can improve the monitoring of operational parameters [15].

The proposed solution uses a Raspberry Pi in combination with an IMU. There are a multitude of devices which were considered and the features of three of them are summarised in Table 1 [16]. All the devices considered are significantly cheaper than IMU motion tracking systems with the cost of some of these devices being over $£ 1000$ [17]. Not only does this cost exclude the cost of installation, these devices are not customised for measuring ship motion. A ship-ready motion measurement system would therefore be significantly more expensive than the quoted price. The Raspberry Pi was chosen due to the combination of its low cost and ability to run multiple programs at the same time, unlike the arduino and gadgeteer which can only run a single program at a time. Even though the Arduino seems to be a more popular option for battery powered applications which aim to minimize size, the Raspberry Pi was chosen due to the possibility of a graphical user interface through the HDMI output.

Besides the devices mentioned in Table 1, smartphones were also considered as possible measurement devices. Work by $\mathrm{McCue}$ indicates that this is also a possible solution. However the Raspberry Pi was chosen over a smartphone because of the ability to easily change the IMU sensor being used as the technology progresses.

\section{RELATED WORKS}

\section{A. Small Craft Motion Program(SCraMP)}

In 2011, the Small Craft Motion Program (SCraMP) [18] was released for the iPhone Operating System (iOS). This application is aimed at providing low budget operators, specifically fishermen, with critical information pertaining to the state of their ship. It makes use of the iPhone's accelerometer, gyroscope, location capabilities and microprocessor. Safety information is presented to the user in a colour-coded graphical format as seen in figure 3 .

In the application, the user can choose which sensor to use, what to display, limits for $\operatorname{roll}(\phi), \operatorname{pitch}(\theta)$, and heave $(z)$, and record and email data. The user can also access the data through iTunes. One particularly interesting feature of SCraMP

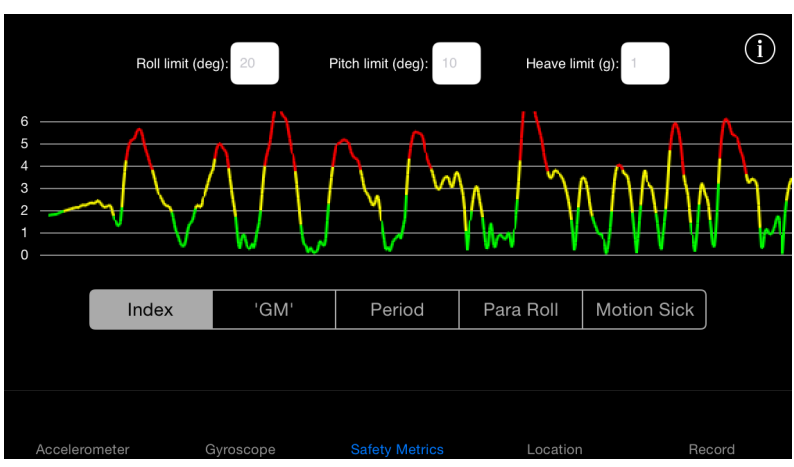

Fig. 3. SCraMP application

is that it uses an index and the roll period method as safety metrics. The index metric is loosely based upon Energy Index research by O'Reilly [19].

$$
\text { Index }=4 \sqrt{\left(\frac{\phi}{\phi_{\text {limit }}}\right)^{2}+\left(\frac{\theta}{\theta_{\text {limit }}}\right)^{2}+\left(\frac{\ddot{z}}{\ddot{z}_{\text {limit }}}\right)^{2}}
$$

The SCraMP application was tested with an iPhone 5 equipped with an STMicroelectronics L3G4200DH 3-axis digital micro-electro-mechanical system (MEMS) gyroscope [20] and the LIS331DLH 3-Axis MEMS accelerometer [21]. The phone also features an AKM magnetometer and Apple's A6 processor. The application allows the user to set the sample rate of recording between 1 and $50 \mathrm{~Hz}$.

This application provides a low-cost method of measuring ship motion. Even though the application is free to purchase in Apple's app store, usage of this application requires the entry cost of an iPhone. Measuring motions at different positions on the vessel simultaneously would require more than one iPhone. Therefore even though this solution is marketed as a low-cost solution, the cost is still relatively high. Additionally one would need to put some effort into making the iPhone waterproof to prevent damage during operation.

\section{B. Xsens MTi sensors}

The MTi sensors produced by XSens have been used by MARIN Wageningen, a leading research institution, to measure ship motion in their Lashing@ Sea project [22]. There are other products on the market similar to this which have also been purposefully built for measuring ship motion such as the Ekinox-A sensor [23] by SBG systems. The MTi 10series gives the systems integrator a choice of three different integration levels (inertial measurement unit, variable reluctance sensor or attitude and heading reference system). These sensors are built specifically for industry and large vessels and offshore structures and therefore have a significantly high cost. This high cost usually includes a software suite, allowing users to easily configure the sensors through an easy-to use graphical interface. However for a small scale fisherman, are all the specifications of a high cost motion measurement unit necessary? For instance, the XSens MTi sensor has an output frequency of up to $2 \mathrm{kHz}$ but for measuring ship motion, 5 $\mathrm{Hz}$ is sufficient [24]. 


\section{Proposed Method}

In this section a description of the proposed approach is provided. The developed solution takes into consideration the advantages and disadvantages of the related work discussed. Firstly, the Raspbian Debian Wheezy operating system was installed on the SD card. A minimum size of 8 Gigabytes is recommended to ensure enough space for all the additional tools needed and storage of the motion data to be recorded. The Raspberry Pi was then connected to the internet and secure shell (SSH) was enabled to allow remote command line access to the Raspberry Pi. Afterwards, the I2C interfaces and automatic loading of the I2C kernel module was enabled. This was necessary because $\mathrm{I} 2 \mathrm{C}$ is the communication protocol used by the IMU sensor.

Building this device required careful consideration of what to install on the OS, how to power the device, how to communicate with and control it, and which sensor module to use. Firstly we only installed needed tools on the Raspberry Pi in order not to have any unnecessary packages that may slow the data collection process down. The following is a list of the packages installed on the pi; python3-dev, python3-setuptools, distribute, python3-pip, plotly, python-smbus, libi2c-dev, i2ctools. An Anker Astro Mini 3200mAh portable charger was used to power the Raspberry Pi. Upon initial testing it powered the device for at least 5 hours. A wireless USB nano wifi dongle with a Ralink RT5370 chipset was used to wirelessly connect the Raspberry Pi to the internet to enable communication via SSH. This wifi dongle can also support access point mode and supports 64/128-bit WEP, WPA/WPA2 encryption methods and $802.1 \mathrm{x}$ authentication standards. The sensor module used was a GY-85 9-axis degree of freedom IMU sensor. This consists of an ITG3205 triple-axis MEMS gyroscope, an ADXL345 MEMS triple-axis accelerometer, and a HMC5883L MEMS triple-axis digital compass. All 3 of these use the $\mathrm{I} 2 \mathrm{C}$ communication protocol, hence why this was enabled earlier. The Raspberry Pi and IMU module were enclosed in a case and the portable charger is connected as shown in figure 4.

A python script was used to continuously collect accelerometer, gyroscope and magnetometer data and save this data to a comma separated value (csv) file. When this sensor was deployed, data was collected at a rate of $50 \mathrm{~Hz}$ during a fishing vessel's operation in Poole Harbour, United Kingdom, over a period of 2 hours. To achieve this, automatic login of the user was setup and the script was added to the profile so that the data collection script would run automatically once the Raspberry Pi had power. In order to accurately make sense of the data, when mounting the Raspberry Pi on the vessel, a note was taken of the axis orientation of the IMU in relation to the vessel so we would know which axis measured which of the vessel's motions. In our execution the $\mathrm{x}$ axis of the IMU was in line with the vessel's lateral axis therefore measuring pitch.

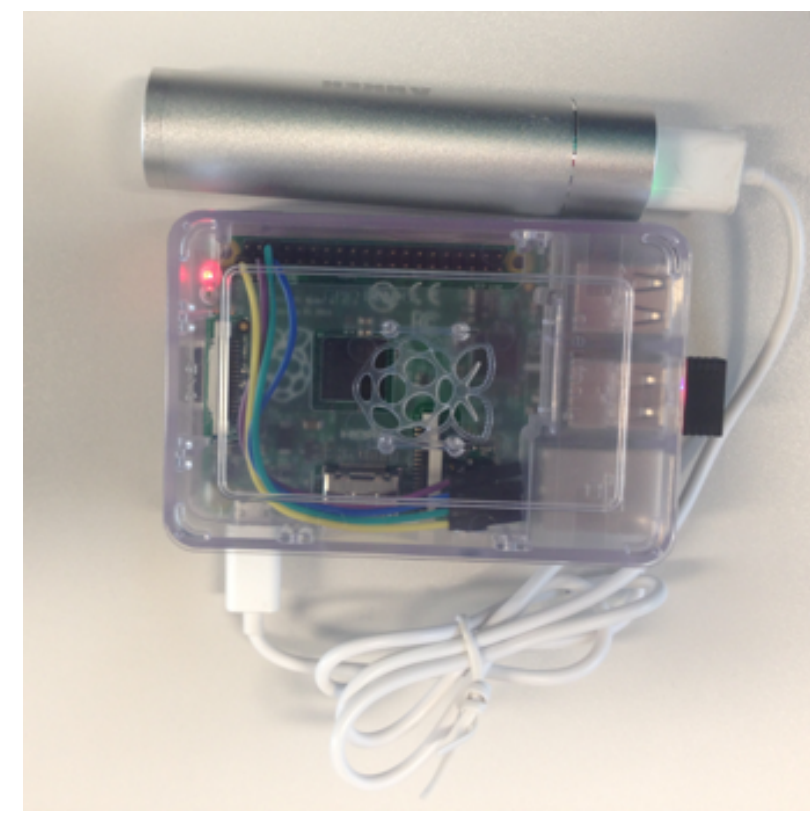

Fig. 4. Motion sensor setup

\section{DISCUSSION}

The Raspberry Pi worked as expected and stored all the data at the expected frequency of $50 \mathrm{~Hz}$. The total length of time over which this data was collected was 2 hours and 7 minutes. Over this period, 20 separate variables were recorded by the Raspberry Pi including time, heading, declination, both scaled and unscaled accelerometer and gyroscope readings in the $\mathrm{X}, \mathrm{Y}$, and $\mathrm{Z}$ axis. The gyroscope has a sensitivity of 14.375 LSBs per degree per second with a full scale range of \pm 2000 degrees per second. The accelerometer has a resolution of $4 \mathrm{mg}$ per LSB, enabling measurement of angles less than 1 degree. The digital compass has a wide magnetic field range of \pm 8 Oe

Figure 5 shows the raw data recorded by the gyroscope in the $\mathrm{x}$ axis over the entire 2-hour period while figure 6 shows only a section of this data between the tenth and eleventh minute of recording. From the 2 graphs it can be seen that the data is noisy but with some filtering this can be improved.

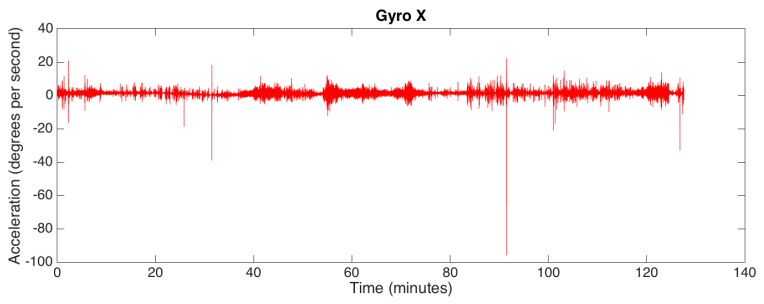

Fig. 5. $\mathrm{X}$ axis gyroscope readings for entire period of recording

Most of the readings from the gyroscope and accelerometer looked similar to the figures shown with all of them having a significant spike between the 91st and 92nd minute of recording when the vessel hit a particularly large wave. 


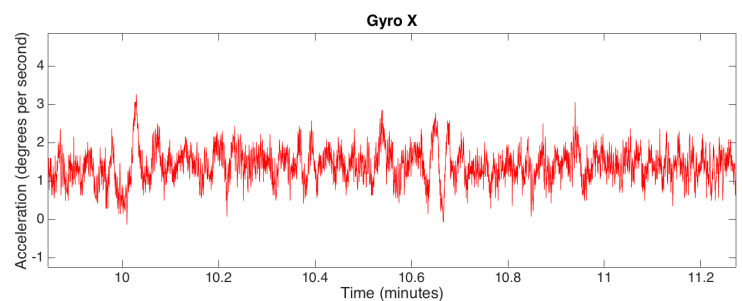

Fig. 6. $\mathrm{X}$ axis gyroscope readings between 10th and 11th minute

As discussed in Section II, measurements of sea surface elevation can be used to obtain the wave spectra. Typically, records of sea surface elevation used in deducing the wave spectra are approximately between $15-20$ minutes with a large enough number of samples being greater than thirty. With respect to wave spectra, the sea builds up from high to low frequency. During the Joint North Sea Wave Observation Project, it was found that the wave spectrum is continuously developing [25]. It is important at this stage to understand how the development of the sea's intensity is represented in the wave spectrum.

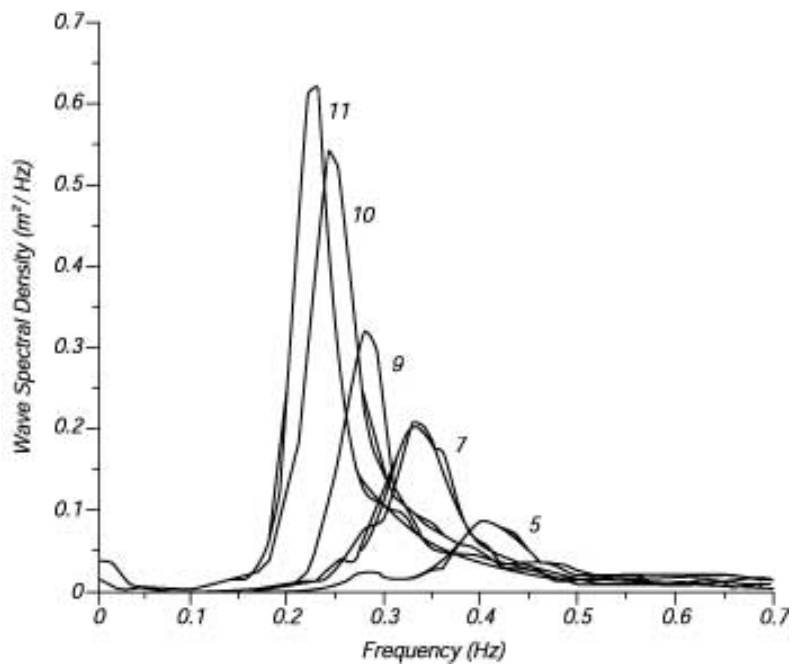

Fig. 7. Wave spectra of developing sea

Figure 7 [25] shows the JONSWAP spectrum for increasing intensities of sea state. This figure shows that the spectrum develops starting from the high frequency to the low frequency with the size of the peak increasing. Therefore from the data, we expect the peak to be at a relatively high frequency (above $0.5 \mathrm{~Hz}$ ) when the time-series is converted to a spectrum since the sea state was particularly calm on the day the data was recorded.

Before obtaining the wave spectrum from the time series, the time series was split into segments, each lasting 20 minutes. This was done because the sea state is assumed to be a stationary process within a time interval of 20 minutes. The power spectral density was then obtained using Welch's method to divide each segment into smaller segments each with a length of 150 data points. Upon converting the data to a spectrum, most of the segments had multiple peaks. However, these peaks were above $5 \mathrm{~Hz}$ and are therefore due to operational conditions such as vibrations where the Raspberry Pi was mounted. Ignoring this part of the spectrum, the results obtained were as expected with the location of the peak above $0.5 \mathrm{~Hz}$ as seen in figure 8 .

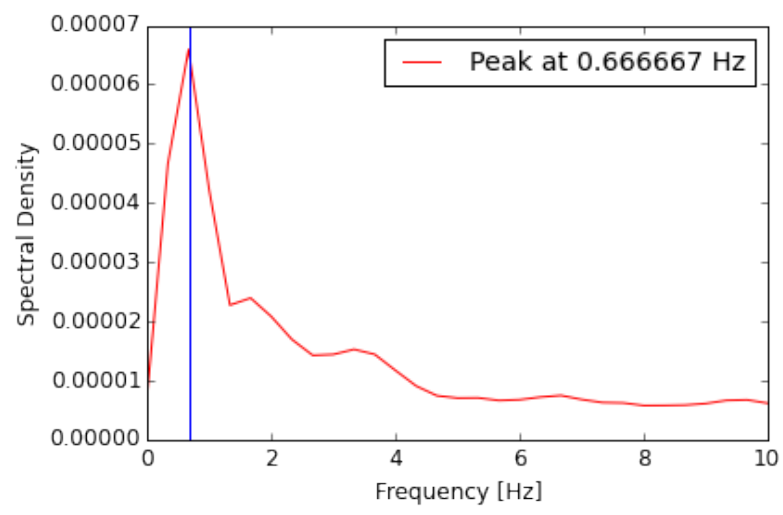

Fig. 8. Spectrum of accelerometer readings

The results obtained show that measurements obtained using this low-cost sensor can be used to obtain relevant knowledge regarding the sea state. Even without compensating for the changing orientation of the vessel with regards to the wave direction and using raw unfiltered data we still obtained a graph of similar shape to what is expected. To improve the quality of information, the readings from the gyroscope and accelerometer should be combined using a filter to obtain measurements for the vessel's translational and rotational degrees of freedom. When represented in the same form as the wave spectrum, these will be the vessel's response amplitude operators and by identifying the transfer function relating these to the wave spectrum, one can deduce the sea state from measured ship motion.

The measured vessel motion can also be used to assess vessel stability. This may be achieved through the wave spectra by using a formulation in the period domain instead of the frequency. Using the period domain has the advantage of being easier to communicate the spectrum to personnel onboard vessels. In addition, information presented in the period domain is much more useful to personnel and has a number of application such as assessing the stiffness of a vessel using the approximate roll period method. The measured motions such as pitch and heave could also be used in calculating an index similar to the one calculated using equation 2 , which will provide vessel operators with knowledge regarding how close they are to their operational limits.

\section{FUTURE WORK}

The development of a low cost sensor will not only improve the operational safety of vessels' but also provide researchers with a useful tool for towing tank tests. This is currently an area of active investigation [26]. In a research environment where there is a higher likelihood of internet access, tools such 
as Plotly, an online data visualization and analytics tool, will enable researchers to easily graph and share data regarding motions of hulls being tested. One could also connect the Raspberry Pi to the IBM internet of Things Foundation for visualization. Alternatively, one could build one's own streaming service, for instance, by using the MEAN (MongoDB, ExpressJs, AngularJS, NodeJS) Stack.

In future work a variety of improvements will be implemented, beginning with the use of a Global Positioning System (GPS) module. This will allow the position of the vessel to be tracked, enabling results obtained to be verified with other data obtained close to the vessel's location such as wave buoys. The use of multiple Raspberry Pis at different points on the vessel will also be investigated to enable the collection of more accurate motion data. In addition, using a Wi-Fi module as an access point will be explored to allow control of the Raspberry $\mathrm{Pi}$. With regards to data collection on the Raspberry $\mathrm{Pi}$, the use of a job scheduler such as Cron will be investigated. Cron will enable the user to schedule exactly when the python script for data collection should be run, in this case, on startup. One advantage of doing this is that the python script will be run in the background, enabling one to log into the Raspberry Pi without interrupting the data collection process.

In the future, information from such a system could be made available freely through automatic identification systems (AIS) used by vessel traffic services (VTS) such as Marine Traffic. These systems provide information on each live vessel such as its type, length, breadth, draught, and speed. In addition, such a system would be able to provide a much finer resolution on a region of interest due to the relatively high number of vessels in service. Marine Traffic has approximately 69,000 live vessels and a total of 508,000 vessels in its database. These numbers are significantly larger than the number of wave buoys deployed worldwide.

\section{CONCLUSION}

In this paper, we presented the use of the Raspberry Pi as low-cost sensor suitable for measuring vessel motions at sea. An evaluation of the proposed method suggest that with further development, the Raspberry Pi can be used to accurately detect the motions of a vessel. In this work, the spectra obtained from the data recorded by the Raspberry $\mathrm{Pi}$ had peak frequencies above $0.5 \mathrm{~Hz}$ as expected for a calm day. Current ways of measuring vessel motion are not only expensive, but also not as easy to install as the solution presented. The use of these sensors will not only provide information relating to the operational safety of vessels but also provide vessel operators with knowledge regarding how vessel motions may change in response to changing conditions.

\section{ACKNOWLEDGMENT}

This work was supported by the UK Engineering and Physical Sciences Research Council for the University of Southampton Faculty of Engineering and the Environment. The data recorded by the Raspberry $\mathrm{Pi}$ is available at http://eprints.soton.ac.uk/id/eprint/383517

\section{REFERENCES}

[1] U. D. Nielsen, "Estimations of on-site directional wave spectra from measured ship responses," Marine Structures, vol. 19, no. 1, pp. 33-69, 2006.

[2] — , "The wave buoy analogy - estimating high-frequency wave excitations," Applied Ocean Research, vol. 30, no. 2, pp. 100-106, 2008

[3] "The most dangerous job in the UK," British Broadcasting Corporation, 2010. [Online]. Available: http://www.bbc.co.uk/news/uk-10923190

[4] S. Almond, "The UK Fishing Industry in 2010," Marine Management Organisation, 2011. [Online]. Available: http://marinemanagement.org. uk/fisheries/statistics/documents/ukseafish/2010/structure_activity.pdf

[5] W. Marx, "America's Most Dangerous Job Is Still Fishing," 2015. [Online]. Available: http://www.bloomberg.com/news/videos/ 2015-05-13/deadly-catch-making-alaska-s-fishing-industry-safer

[6] B. Barrass and C. Derrett, Ship stability for masters and mates, sixth edition ed. Elsevier Ltd, 2006.

[7] H. Enshaei, "Prevention of extreme roll motions through measurements of ship's motion responses," Ph.D. dissertation, Newcastle University, 2013.

[8] The Met Office, "Wavenet and HF radar." [Online]. Available: http://research.metoffice.gov.uk/research/ocean/wavenet/index.html

[9] US Army Corps of Engineers, "Coastal Engineering Manual," 2002. [Online]. Available: http://www.publications.usace.army.mil/Portals/76/ Publications/EngineerManuals/EM $\left\{\backslash_{-}\right\} 1110-2-1100\left\{\backslash_{-}\right\}$Part-02.pdf

[10] W. H. Michel, "Sea Spectra Simplified," Marine Technology, vol. 5, no. 1 , pp. 17-30, 1968.

[11] H. E. Krogstad and Ø. A. Arntsen. (2000, February) Linear wave theory.

[12] L. Razdolsky, Probability-Based Structural Fire Load. Cambridge University Press, 2014, no. 9781107038745.

[13] National Oceanic and Atmospheric Administration UCAR Comet Program, "Wave statistics," 2009.

[14] International Telecommunication Union, "Overview of the Internet of things," Recommendation ITU-T Y.2060, 2012.

[15] M. Giacobbe, E. Guglielmino, and A. Puliafito, "A platform for integrated data processing from heterogeneous devices in naval field," in Proceedings - International Symposium on Computers and Communications, 2009, pp. 520-525.

[16] A. Wong, "Prototyping Connected-Devices for the Internet of Things." [Online]. Available: http://www.isoc.hk/wp-content/uploads/ 2013/08/Angus-Wong-OUHK-Prototyping-Tools-for-IoT.pdf

[17] F. element14, "XSENS MTI-30-2A5G4 MEMS Module." [Online]. Available: http://uk.farnell.com/xsens/mti-30-2a5g4/ ahrs-mems-motion-tracking-module/dp/2452308

[18] L. McCue, "Putting vessel motion research into the hands of operators," in Proc. 11th International Conference on the Stability of Ships and Ocean Vehicles, Athens, Greece, September 2012.

[19] P. J. F. O'Reilly, “Aircraft/Deck Interface Dynamics for Destroyers," Marine Technology, vol. 24, no. 1, pp. 15-25, 1987.

[20] "L3G4200D MEMS motion sensor: three-axis digital output gyroscope datasheet," ST Microelectronics Inc, 2010. [Online]. Available: http: //www.digchip.com/datasheets/parts/datasheet/456/L3G4200D-pdf.php

[21] "LIS331DL MEMS motion sensor 3-axis - 2g/8g smart digital output accelerometer datasheet," ST Microelectronics Inc, 2007. [Online]. Available: http://www.digchip.com/datasheets/parts/datasheet/ 456/LIS331DL-pdf.php

[22] J. Koning, "Lashing@Sea executive summary," Marin, 2009. [Online]. Available: \{http://www.marin.nl/web/JIPs-Networks/ Archived-JIPs-Public/Lashingsea.htm $\}$

[23] "Ekinox Series; Tactical Grade MEMS Inertial Systems," SBG Systems, 2014. [Online]. Available: http://www.sbg-systems.com/docs/Ekinox_ Series_Leaflet.pdf

[24] "Motion monitoring," Maritime Research Institute Netherlands, 2012. [Online]. Available: http://www.marin.nl/web/Research-Topics/ Offshore-operations/Motion-monitoring.htm

[25] K. Hasselmann, T. Barnett, E. Bouws, H. Carlson, D. Cartwright, K. Enke, J. Ewing, H. Gienapp, D. Hasselmann, P. Kruseman, A. Meerburg, P. Mller, D. Olbers, K. Richter, W. Sell, and H. Walden, "Measurements of wind-wave growth and swell decay during the joint north sea wave project (jonswap)," Ergänzungsheft zur Deutschen Hydrographischen Zeitschrift Reihe, no. 12, p. 95, 1973.

[26] S. Bennet, C. Brooks, B. Winden, D. Taunton, A. Forrester, S. Turnock, and D. Hudson, "Measuring of ship hydroelastic response using multiple wireless sensor nodes," Ocean Engineering, 2014. 